with recruitment in two phases; contacting Directors of Public Health (DsPH) directly and snowballing through key contacts. Other professionals were interviewed where recommended by DsPH. Face-to-face or telephone interviews were digitally recorded and transcribed verbatim, and data analysed using the framework method.

Results Public health professionals $(n=14)$ in ten LAs were interviewed, including DsPH $(n=5)$ and consultants $(n=5)$. Participants viewed evidence use as an important part of their roles, and had a high level of relevant skills. However, public health teams and individuals differed in the extent to which they used systematic reviews. Some consultants used systematic reviews extensively; for other participants, while they were highly valued in principle, their value and impact in practice was limited by various barriers and the nature of decisionmaking in LAs. Barriers included time constraints, a lack of relevant reviews, limited applicability to LA context and the role of evidence in a political organisation. Evidence, including systematic reviews, was sometimes used to directly inform decisions but also used tactically. Participants regularly engaged with research evidence outside the decision-making process, often through a personal commitment to maintaining knowledge.

Conclusion Systematic reviews are used in LA public health in various ways but use varies and is limited by a range of factors. Decision-making processes can be complex, with systematic reviews used in conjunction with other evidence types, and therefore research use should be seen within the political context. This qualitative study developed understanding of systematic review use but a limitation is that there may be professionals less committed to evidence use that were not included in the sample.

\section{OP93 THE MENTAL HEALTH IMPACT OF UNIVERSAL CREDITINTRODUCTION ON WORKING AGE UNEMPLOYED INDIVIDUALS ACROSS ENGLAND: DIFFERENCE-IN-DIFFERENCE ANALYSIS OF THE UNDERSTANDING SOCIETY HOUSEHOLD LONGITUDINAL STUDY}

SL Wickham*, L Bentley, T Rose, D Taylor-Robinson, B Barr. Public Health and Policy, University of Liverpool, Liverpool, UK

\subsection{6/jech-2019-SSMabstracts.97}

Background An increase in mental health problems and a widening in inequalities has occurred in England during a period of austerity and welfare reform. Universal Credit (UC) was introduced for unemployed people first in the UK in 2013, to simplify the benefit system by bringing six 'legacy benefits' together into one means tested benefit. Qualitative research suggested potential negative mental health impacts. We assessed this natural policy experiment, taking advantage of the variation in UC introduction across local authority areas to see whether the introduction of UC was associated with an increase in self-reported psychological distress.

Methods We conducted a difference-in-difference analysis using longitudinal data from the Understanding Society Household Panel Survey on 88,060 working age (16-64) adults who participated between 1991 and 2018. We linked UC data from the DWP by local authority, month and year to identify when UC was introduced in each area. The primary outcome was self-reported psychological distress using the continuous
General Health Questinaaire-12 (GHQ-12) measure, with high scores indicating increased psychological distress. We compared the change in mental health of unemployed people after UC was introduced in their local authority area to individuals who were not unemployed, and not effected by UC. Analysis was conducted in STATA-14.2 and accounted for confounding variables such as year, age, sex, having dependents, and marital status.

Results $18 \%(\mathrm{n}=15,847)$ had a spell of unemployment at some time between 1991 and 2018. 3058 of these were unemployed in an area where UC had been introduced. When UC was introduced in an area the mental health of the unemployed deteriorated, whilst we did not see a deterioration in the mental health of those not unemployed. The trends in mental health in these two groups prior to the introduction of UC were parallel. The difference-in-differences in total GHQ-12 scores showed that the introduction of UC on an area was associated with a rise in psychological distress amongst the unemployed $(\beta=0.27,95 \%$ CI $0.09,0.45$, $\mathrm{p}=.003)$ relative to those not unemployed.

Conclusion Our analysis shows that the introduction of UC has led to an increase in psychological distress amongst unemployed individuals. UC has the potential to positively transform the benefit system, however our analysis, alongside the growing body of evidence suggests that in its current form UC has a negative impact on peoples mental health, and actions to address this are needed to help tackle the UK mental health crisis.

\section{OP94 HEALTH AND PRODUCTIVITY COSTS OF VIOLENCE AGAINST WOMEN AND GIRLS}

${ }^{1} \mathrm{~N}$ Durvury, ${ }^{1} \mathrm{M}$ Chadha*, ${ }^{1} \mathrm{~S}$ Scriver, ${ }^{2} \mathrm{~S}$ Raghavendran, ${ }^{3} \mathrm{~F}$ Asante, ${ }^{4} \mathrm{~K}$ Ghaus, ${ }^{5} \mathrm{~K}$ Elmusharaf, ${ }^{1} \mathrm{M}$ Sabir, ${ }^{1} \mathrm{G}$ Mcdarby, ${ }^{1} \mathrm{C}$ Ballantine. ${ }^{1}$ Political Science and Sociology, NUI Galway, Galway, Ireland; '2J.E. Cairnes School of Business and Economics, NUI Galway, Galway, Ireland; '3nstitute of Statistical, Social and Economic Research, University of Ghana, Accra, Ghana; ${ }^{4}$ Social Policy and Development Centre, Karachi, Pakistan; ${ }^{5}$ Graduate Entry Medical School, University of Limerick, Limerick, Ireland

\subsection{6/jech-2019-SSMabstracts.98}

Background Violence against Women and Girls (VAWG) is widely recognized as a violation of human rights and a challenge to public health. In recognition of the dearth of knowledge of the impacts and costs due to VAWG, particularly in fragile and developing contexts, the UK Department for International Development (DFID) funded this research to investigate the social and economic costs of VAWG in Ghana, Pakistan and South Sudan (2014-2019), as part of its wider What Works to Prevent Violence research and innovation programme.

Methods This study used a mixed method approach including both quantitative surveys of individual women, households, and businesses, and qualitative inquiry methods including key informant interviews, participatory focus groups, and individual in-depth interviews. The direct health-related effects on women are measured by the out of pocket health expenditure incurred by women experiencing violence. The indirect mental health-related effects are quantified by productivity loss which is estimated as women missing work, being late at work or being not being mentally present at work. The study also estimates various health-related scores: Depression, Disability and Acute Illness scores, and compares women who experience violence and who do not. 
Results In all the three countries, this study finds health expenditure to be the predominant out of pocket cost incurred by women experiencing violence. Women who experience violence also have statistically significant higher depression, disability and acute illness scores, and thus indicate the broader health impacts of VAWG. These health impacts affect the overall productivity of women experiencing violence. Approximately 80 million productivity work days in Pakistan, 65 million productivity work days in Ghana, and 8.5 million productivity work days in scaled population of South Sudan are lost due to women experiencing any violence. The productivity loss indicates the significant impact VAWG has on the overall economy.

Conclusion The results of this study on the socioeconomic cost of VAWG highlight the need for crucial action by a wide range of actors, from local authorities and community leaders to national government. Moreover, the results suggest the potential burden that VAWG places on the health sector in the countries studied. The health and economic impacts outlined in this study together build a strong economic case for investment by government and donors in the prevention of VAWG.

\section{OP97 DISPARITIES IN BREAST CANCER SCREENING UPTAKE ACCORDING TO TYPE OF MENTAL ILLNESS: A POPULATION-BASED RECORD LINKAGE STUDY}

${ }^{1} \mathrm{E}$ Ross ${ }^{*},{ }^{1} \mathrm{~A}$ Maguire, ${ }^{1} \mathrm{M}$ Donnelly, ${ }^{2} \mathrm{~A}$ Mairs, ${ }^{2} \mathrm{C}$ Hall, ${ }^{1} \mathrm{D}$ O'Reilly. ${ }^{1}$ Centre for Public Health, Queen's University Belfast, Belfast, UK; ${ }^{2}$ Public Health Agency, Belfast, UK

\subsection{6/jech-2019-SSMabstracts.99}

Background Evidence from the United States (US) suggests that uptake of breast cancer screening is lower for women with severe mental illness, but it is unclear whether this applies to the United Kingdom (UK) where services are free at the point of access. Furthermore, no attempt has been made to investigate whether this finding extends to the more prevalent mental disorders. The aim of this study was to conduct the first population-wide record linkage study of the influence of poor mental health and different types of mental disorder (as assessed by use of psychotropic medications) on routine breast screening uptake in the UK.

Methods Breast screening records were obtained from the National Breast Screening System (NBSS) and were subsequently linked to 2011 Census data within the Northern Ireland Longitudinal Study (NILS). This resulted in a cohort of 57,328 women who were followed through one complete three-year screening cycle of the National Health Service (NHS) breast screening programme. Psychotropic medication receipt was derived from a centralised prescribing database and classified according to prescriptions in the three months preceding screening invite. Other individual and householdlevel cohort attributes known to be associated with breast screening uptake were derived from Census records. Logistic regression was employed to calculate age-only and fullyadjusted odds ratios (ORs) and 95\% confidence intervals of attendance at breast screening.

Results Over a third of women received at least one prescription for psychotropic medication in the three months prior to screening invite and these women were $15 \%$ less likely to attend screening (OR 0.85: 0.81-0.88). Although significantly reduced uptake was observed for individuals in receipt of all types of psychotropic medication, attendance was particularly low for women prescribed antipsychotics (OR 0.63: 0.560.70 ), anxiolytics (OR 0.61: 0.57-0.66), or hypnotics (OR 0.68: 0.63-0.72). Additionally, there was evidence that this association was further influenced by severity of mental illness (as assessed by duration of medication usage).

Conclusion This study advances our current understanding of the factors contributing to suboptimal breast screening uptake rates, confirming the existence of disparities in breast screening uptake for individuals with poor mental health in the United Kingdom (U.K), and for the first time, demonstrating that the observed disparities vary according to the type and severity of mental disorder examined. The extension of this association to common mental disorders is of particular concern given the high prevalence of these disorders worldwide.

\section{OP111 ORAL HEALTH, DISABILITY AND PHYSICAL FUNCTION: RESULTS FROM STUDIES OF OLDER PEOPLE IN THE UK AND USA}

${ }^{1}$ E Kotronia*, ${ }^{2} \mathrm{GS}$ Wannamethee, ${ }^{2} \mathrm{OA}$ Papacosta, ${ }^{3} \mathrm{PH}$ Whincup, ${ }^{2} \mathrm{LT}$ Lennon, ${ }^{4} \mathrm{M}$ Visser, ${ }^{5}$ RJ Weyant, ${ }^{6}$ TB Harris, ${ }^{1,2}$ SE Ramsay. ${ }^{1}$ Institute of Health and Society, Newcastle University, Newcastle Upon Tyne, UK; ${ }^{2}$ Department of Primary Care and Population Health, UCL, London, UK; ${ }^{3}$ Population Health Research Institute, St George's University of London, London, UK; ${ }^{4}$ Department of Health Sciences, Vrije Universiteit, Amsterdam, Netherlands: ${ }^{5}$ Department of Dental Public Health, School of Dental Medicine, University of Pittsburgh, Pittsburgh, USA; ${ }^{6}$ Laboratory of Epidemiology and Population Sciences, National Institute of Aging, Bethesda, USA

\subsection{6/jech-2019-SSMabstracts. 100}

Background Disability and poor physical function have major impacts on the health and well-being of ageing populations. Poor oral health (tooth loss, periodontal (gum) disease, dryness of mouth) are also very common health problems in older populations, and adversely impact nutrition and quality of life. Studies suggest that poor oral health in older age is associated with disability, however most studies have limited oral health measures. We investigated the association of a range of objectively and subjectively assessed oral health markers with disability and physical function in two population-based studies of older people in the UK and USA.

Methods Cross-sectional analyses were conducted in the British Regional Heart Study (BRHS) comprising men aged 71-92 $(n=2147)$ from 24 British towns, and the US Health, Aging and Body Composition (HABC) Study comprising men and women aged 71-80 $(n=3075)$. Assessments included objective measures of oral health (periodontal disease, tooth count), and subjective measures (dry mouth, self-reported oral health, dental service use), and disability [mobility limitations, Activities of Daily Living (ADL) and Instrumental Activities of Daily Living (IADL)], and physical function (grip strength, gait speed, chair stand test). Logistic regression models, adjusted for confounding variables, were used to examine the associations between oral health and disability and physical function. Results In the BRHS, dry mouth, tooth loss, and cumulative oral health problems ( $\geq 3$ problems) were associated with greater risks of mobility limitations, problems with ADL and IADL; these remained significant after adjustment for confounding variables (for $\geq 3$ dry mouth symptoms, odds ratio $(\mathrm{OR})=2.68, \quad 95 \% \mathrm{CI}=1.94-3.69 ; \quad \mathrm{OR}=1.76, \quad 95 \% \mathrm{CI}=1.15$ 2.69; $\mathrm{OR}=2.90,95 \%$ CI: 2.01, 4.18, respectively). Similar results were observed in the HABC Study for mobility limitations and $\mathrm{ADL}$ (for $\geq 3$ oral health problems, $\mathrm{OR}=2.19,95 \%$ 